\title{
Orbitofrontal Dysfunction Related to Both Apathy and Disinhibition in Frontotemporal Dementia
}

\author{
Frédéric Peters $^{a}$ Daniela Perani ${ }^{b}$ Karl Herholz ${ }^{c}$ Vjera Holthoff ${ }^{d}$ \\ Bettina Beuthien-Baumann ${ }^{\mathrm{e}}$ Sandro Sorbi ${ }^{\mathrm{f}}$ Alberto $\mathrm{Pupi}^{\mathrm{g}}$ \\ Christian Degueldre $^{a}$ Christian Lemaire $^{a}$ Fabienne Collette $^{a}$ Eric Salmon $^{a}$ \\ ${ }^{a}$ Cyclotron Research Center, University of Liège, Liège, Belgium; ${ }^{b}$ Vita Salute San Raffaele University, and \\ IRCCS H San Raffaele, IBFM-CNR, Milan, Italy; ${ }^{\circ}$ Department of Neurology, University of Cologne, and \\ Max-Planck Institute for Neurological Research, Cologne, dDepartment of Psychiatry and Psychotherapy, \\ Dresden University of Technology, ${ }^{e}$ Department of Nuclear Medicine, Dresden University of Technology, and \\ PET Center, Research Center Rossendorf, Dresden, Germany; ${ }^{f}$ Department of Neurological and Psychiatric \\ Sciences, University of Florence, ${ }^{9}$ Department of Physiopathology, University of Florence, Florence, Italy
}

\section{Key Words}

Frontotemporal dementia $\cdot$ Positron emission tomography · Apathy · Disinhibition - Social conduct

\begin{abstract}
Orbitofrontal metabolic impairment is characteristic of the frontal variant of frontotemporal dementia (fv-FTD), as are early changes in emotional and social conduct. Two main types of behavioral disturbances have been distinguished in fv-FTD patients: apathetic and disinhibited manifestations. In this study, we searched for relationships between brain metabolism and presence of apathetic or disinhibited behavior. Metabolic activity and behavioral data were collected in $41 \mathrm{fv}$-FTD patients from European PET centers. A conjunction analysis of the PET data showed an expected impairment of metabolic activity in the anterior cingulate, ventromedial and orbital prefrontal cortex, the dorsolateral prefrontal cortex and the left anterior insula in fv-FTD subjects compared to matched controls. A correlation was observed between disinhibition scores on the Neuropsychiatric Inventory
\end{abstract}

scale and a cluster of voxels located in the posterior orbitofrontal cortex $(6,28,-24)$. Comparison of brain activity between apathetic and nonapathetic fV-FTD patients from two centers also revealed a specific involvement of the posterior orbitofrontal cortex in apathetic subjects (4, $22,-22)$. The results confirm that the main cerebral metabolic impairment in fV-FTD patients affects areas specializing in emotional evaluation and demonstrate that decreased orbitofrontal activity is related to both disinhibited and apathetic syndromes in fv-FTD.

Copyright (C) 2006 S. Karger AG, Basel

\section{Introduction}

Frontotemporal dementia (FTD) is one of the major causes of early-onset degenerative dementia. Clinical manifestations are classically characterized by the very evident alteration of personal and social judgment. However, FTD is a heterogeneous pathology, from both a clinical and a neuropathological point of view [1-3]. Three different syndromes are considered to be variants of the

\section{KARGER \\ Fax +4161306 1234 E-Mailkarger@karger.ch} www.karger.com
(C) 2006 S. Karger AG, Basel $1420-8008 / 06 / 0216-0373 \$ 23.50 / 0$

Accessible online at:

www.karger.com/dem
Frédéric Peters

Cyclotron Research Center (Bat. B30), University of Liège

Allée du 6 août 8

BE-4000 Liège (Belgium)

Tel. +32 436623 06, Fax +32 436628 08, E-Mail Frederic.Peters@ulg.ac.be 
Table 1. Participant demographic and behavioral data

\begin{tabular}{llllll}
\hline Group & Participants & Age, years & Males/females & CDR & Duration, months \\
\hline FTD & 23 & $63.5(8.1)$ & $13 / 10$ & $1.5(0.8)$ & $38.9(27.3)$ \\
& 41 & $64.5(8.3)$ & $21 / 20$ & $1.2(0.7)$ & $33.4(22.8)$ \\
Control & 23 & $64.0(7.8)$ & $14 / 9$ & - & - \\
\hline
\end{tabular}

Values for age, CDR and duration are expressed as mean (standard deviation).

disease: semantic dementia, primary progressive aphasia and the frontal variant of FTD (fv-FTD). Structural and functional neuroimaging studies of FTD phenotypes have explored which kinds of brain damage are shared by or specific to the subgroups of FTD. Common involvement of the frontal and insular cortices has been found for all variants of FTD [4]. In semantic dementia, characterized by a progressive loss of semantic knowledge [5, 6], prominent cerebral atrophy has been observed in the anterior temporal cortex and anterior hippocampus [711]. The insula is characteristically involved in patients suffering from primary progressive aphasia (the nonfluent aphasic variant of FTD), who are clinically typified by the production of hesitant and nonfluent speech [12, 13]. Predominant frontal involvement has been observed in the frontal variant of the disease - the most frequent variant - which is clinically characterized by early changes in emotional and social conduct $[10,14]$. There is considerable overlap between the clinically characterized semantic dementia and fv-FTD, and the anatomically defined (i.e., by atrophy) temporal and frontal variants of FTD, respectively [15].

Although major behavioral disorders have been described in the temporal variant of FTD [15], we focus our analysis on clinically defined fv-FTD. In a multicenter study of $29 \mathrm{fv}$-FTD patients, hypometabolic areas common to all patients mainly comprised the ventromedial part of the prefrontal cortex [16]. Moreover, progression of the disease was essentially accompanied by a decrease in metabolic activity in the orbitofrontal region [17]. This cortex is important for the processing of emotional stimuli and the adaptation of behavior according to social rules. However, few studies have explored the neural correlates of clinical phenotypes among fv-FTD patients. Indeed, two main types of social/emotional misconduct have been reported in fv-FTD patients' daily behavior: (1) disinhibition, referring to the production of socially inappropriate comments and/or actions, and (2) apathy, referring to lack of initiative, lack of interest and lack of emotional concern [18]. In the neuroimaging literature, apathy and disinhibition are characterized by impairment of, respectively, the dorsolateral versus orbital frontal metabolism [19], frontopolar versus posterior orbital frontal activity [20], and anterior and dorsolateral prefrontal versus posterior orbitofrontal glucose uptake [21].

In the study reported on here, we evaluated brain metabolic impairment in fv-FTD patients included in a prospective European multicenter study. There were two differences compared to our previous study [16]. First, we used more recent diagnostic criteria [2] than in the previous report [22]. Secondly, we collected behavioral data in order to investigate the relationships between key clinical variables and cerebral metabolism in fv-FTD. We planned to explore three clinical variables in this context: the severity of the dementia, as measured by the Clinical Dementia Rating (CDR) scale [23], apathy and disinhibition, both measured with the Neuropsychiatric Inventory (NPI) [24].

\section{Method}

\section{Patients}

Images obtained with positron emission tomography and the [18F]fluorodeoxyglucose method (FDG-PET) and clinical data were collected in a population of $41 \mathrm{fv}$-FTD patients diagnosed according to international clinical criteria [2]. Patients with progressive aphasia and semantic dementia were not included. The data were gathered in a prospective multicenter European study (Network for Efficiency and Standardization of Dementia Diagnosis or NEST-DD project). These 41 patients were selected from five different PET centers. Their mean age was $63.5 \pm 8.1$ years, mean Mini-Mental State Examination (MMSE) score was $22 \pm 5$, mean CDR was $1.5 \pm 0.8$, and mean disease duration was $39 \pm 27$ months. Data from two, three or five centers were used for different analyses, depending on the availability of control subjects, or according to the distribution of behavioral symptoms at each center. Out of the whole patient group, $23 \mathrm{fv}$-FTD subjects could be age matched to elderly controls from their own center; metabolic differences between the two populations were then confirmed in a 
Table 2. NPI scores for $41 \mathrm{fv}-F T D$ patients

\begin{tabular}{llllllllll}
\hline Apa & Dis & Abe & Agi & Dys & Irr & Anx & Del & Eup & Hal \\
\hline $5.4(4.7)$ & $1.6(2.8)$ & $2.1(3.4)$ & $2.0(3.0)$ & $2.1(3.3)$ & $1.9(3.0)$ & $1.8(3.2)$ & $0.5(1.5)$ & $0.7(2.2)$ & $0.2(0.7)$ \\
\hline
\end{tabular}

Subscales for Apathy (Apa), Disinhibition (Dis), Aberrant motor behavior (Abe), Agitation (Agi), Dysphoria (Dys), Irritability (Irr), Anxiety (Anx), Delusion (Del), Euphoria (Eup), Hallucination (Hal). Scores are expressed as mean (standard deviation).

conjunction study of data from those (three) centers (see table 1 for demographic data). The controls had no history of neuropsychiatric problems, memory disorders or drug abuse. The brain CT or MRI data were visually analyzed for each subject in each center: none had either a focal vascular lesion or major cerebral atrophy. Written and informed consent was obtained from all the participants in this study according to local Ethics Committee requirements.

\section{Behavioral Measures}

We used a measure of dementia severity, the CDR [23], and the NPI [24]. We were particularly interested in two subscales of the NPI: Apathy and Disinhibition. Clinical data on our fv-FTD patients are summarized in tables 1 and 2.

\section{PET Acquisition and Image Processing}

Basic images were acquired during quiet wakefulness with eyes closed and ears unplugged after intravenous injection of 110 $370 \mathrm{Mbq}{ }^{18} \mathrm{~F}$-2-fluoro-2-deoxy- $D$-glucose. Images of tracer distribution in the brain were used for analysis; the required minimum scan starting time was $30 \mathrm{~min}$ after tracer injection and scan duration was approximately $20 \mathrm{~min}$. Images were reconstructed using filtered backprojection including correction for measured attenuation and scatter using the standard software supplied by the various scanner manufacturers [25].

SPM2 routines (Wellcome Department of Cognitive Neurology, London, UK) implemented in MATLAB (Mathworks Inc., Sherborn, Mass., USA) were used to perform basic image processing and voxel-based statistical analysis. In the coordinating center (Cologne), all PET scans were checked and spatially normalized by nonlinear and affine 12-parameter transformations to the SPM2 standard brain template. Then images were transferred to the FTD task force center (Liège) and smoothed with a 12-mm FWHM isotropic kernel. Metabolic changes in fv-FTD patients were estimated according to a general linear model using linear contrasts. Global activity adjustment was performed using proportional scaling.

\section{Data Analysis}

First of all, a comparison of brain activity was conducted in order to delineate common hypometabolic areas in our fv-FTD population. Twenty-three patients from three centers were matched by age to a control from their own center. Thus, 23 pairs of scans were used in a multigroup experimental design (three groups from three PET centers involved in the NEST-DD project) with two conditions (considering each pair of scans as a subject, and treating FTD as condition 1 and controls as condition 2). A comparison of brain metabolism was computed between patients and controls from each center. Then a conjunction analysis using data from the three centers was carried out. This was a confirmatory analysis $[16,17$, 21 ], and a threshold of significance was fixed to $\mathrm{p}$ (uncorrected) $<$ 0.01 . A masking procedure was applied to ensure that the contrasts between FTD and control subjects in each center were taken into account as an inclusive mask, with a mask $\mathrm{p}$ value $<0.01$. In that way, we focused exclusively on metabolic impairments common to all three PET centers.

At this point, we did two different statistical analyses to investigate the relationship between FTD metabolism and clinical variables. First, we performed a correlation analysis between the behavioral data collected in the whole FTD group $(n=41)$ and PET metabolic measurements. Three different variables were used for correlation analysis: dementia severity (CDR score), and NPI scores of apathy and disinhibition (design: single subject, covariates only, with age as a confounding covariate). The correlated set of clusters was thresholded at $\mathrm{p}$ (uncorrected) $\leq 0.001$.

We then performed direct comparison analyses in order to replicate previous findings [21] by comparing metabolism in the different behavioral subgroups of fv-FTD. We therefore divided the FTD group into subgroups based on NPI scores for disinhibition and apathy. A score of 8 or more was considered to indicate pathology while a score of 4 or less was considered to indicate a preserved capacity. Patients with both disinhibition and apathy were excluded from the analysis. The samples of disinhibited FTD patients were too small within each center to perform any analysis. According to the NPI apathy scores, 13 FTD patients from two centers were classified as apathetic (mean NPI apathy score: 9.8 ) whereas 12 FTD patients from the same centers were considered as 'nonapathetic' (mean NPI apathy score: 1.5). A single-subject experimental design was used in the SPM software, with six conditions, treating each subgroup from centers 1 (Milan) and 2 (Liège) as a different condition [apathetic FTD (1); nonapathetic FTD (1); elderly controls (1); apathetic FTD (2); nonapathetic FTD (2) and elderly controls (2)]. Age and center were introduced as confounding covariates. We compared the metabolism of each apathetic FTD group to the entire control group from their own center. Then, a conjunction analysis of these comparisons (apathetic FTD vs. controls) was carried out with a $\mathrm{p}$ (uncorrected) $\leq 0.001$ and a masking procedure excluding the hypometabolic areas observed in nonapathetic FTD patients (exclusive mask: nonapathetic FTD vs. controls at $p$ value $<0.05$ ). In that way, we wished to isolate the hypometabolic areas specific to apathetic FTD in the two centers, and not shared by nonapathetic FTD patients. For all analyses, brain coordinates for the SPM results corresponded to the MNI standard space. 
Fig. 1. Correlation analysis between NPI disinhibition scores and metabolic images in $41 \mathrm{fv}$-FTD subjects. a Correlation in the gyrus rectus. b Correlation between disinhibition scores of all subjects (x-axis) and the relative metabolic activity in the gyrus rectus (y-axis).

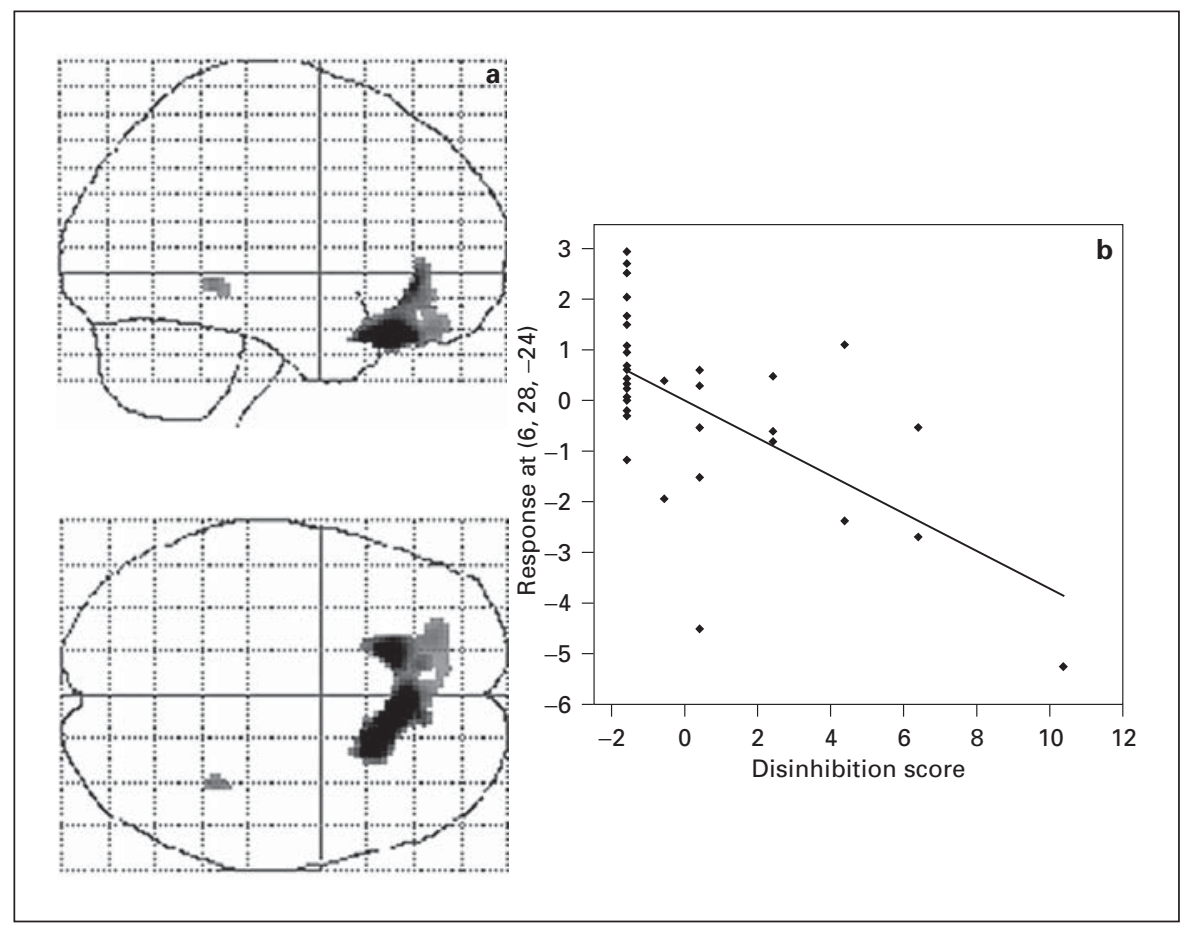

Table 3. Hypometabolism in FTD

\begin{tabular}{|c|c|c|c|c|c|c|c|}
\hline & \multirow[t]{2}{*}{ Brain structure } & \multirow{2}{*}{$\begin{array}{l}\text { Hemi- } \\
\text { sphere }\end{array}$} & \multicolumn{3}{|c|}{ Coordinates } & \multirow{2}{*}{$\begin{array}{l}\mathrm{Z} \\
\text { value }\end{array}$} & \multirow{2}{*}{$\begin{array}{l}\text { Voxel } \\
\text { extent }\end{array}$} \\
\hline & & & $\mathrm{x}$ & $\mathrm{y}$ & $\mathrm{z}$ & & \\
\hline \multirow[t]{11}{*}{ Conjunction analysis } & Medial frontal cortex & & & & & & \multirow{6}{*}{1,656} \\
\hline & Anterior cingulate & $\mathrm{L}$ & -12 & 42 & 14 & 3.76 & \\
\hline & \multirow[t]{2}{*}{ Superior frontopolar gyrus } & $\mathrm{L}$ & -16 & 64 & 20 & 2.74 & \\
\hline & & $\mathrm{R}$ & 12 & 60 & 26 & 2.89 & \\
\hline & \multirow[t]{2}{*}{ Superior frontal gyrus } & $\mathrm{L}$ & -32 & 40 & 32 & 2.48 & \\
\hline & & $\mathrm{R}$ & 24 & 42 & 38 & 3.14 & \\
\hline & Lateral frontal cortex & & & & & & \\
\hline & Inferior prefrontal gyrus & $\mathrm{L}$ & -46 & 12 & 34 & 2.91 & 162 \\
\hline & Anterior insula & $\mathrm{L}$ & -44 & 20 & 2 & 2.56 & 44 \\
\hline & Orbitofrontal cortex & & & & & & \\
\hline & Gyrus rectus & $\mathrm{L} / \mathrm{R}$ & -4 & 44 & -22 & 2.80 & 208 \\
\hline Correlation with disinhibition & Gyrus rectus & $\mathrm{L} / \mathrm{R}$ & 6 & 28 & -24 & 4.15 & 886 \\
\hline Apathetic vs. nonapathetic & Gyrus rectus & $\mathrm{L} / \mathrm{R}$ & -4 & 22 & -22 & 3.21 & 182 \\
\hline
\end{tabular}

Results are reported in MNI spatial coordinates and are expressed as $\mathrm{x}, \mathrm{y}$ and $\mathrm{z}(\mathrm{mm})$.

\section{Results}

The patterns of hypometabolism obtained by the comparison of fv-FTD patients and matched controls were very similar in the three selected centers. The conjunction analysis carried out on these comparisons showed decreased activity in the left anterior cingulate $(-12,42,14)$, the ventromedial and orbital prefrontal cortex, the left anterior insula and different areas of the lateral prefrontal cortex (LPFC; table 3). 
Fig. 2. Comparison between the metabolism of apathetic FTD patients and controls from their own center with an exclusive masking procedure to exclude hypometabolic areas of the nonapathetic FTD group. a Specific hypometabolism in the gyrus rectus of apathetic FTD group. b Design matrix; age and center were taken as confounding covariates. (1) apathetic FTD (center 1); (2) nonapathetic FTD (center 1); (3) control subjects (center 1); (4) apathetic FTD (center 2); (5) nonapathetic FTD (center 2); (6) control subjects (center 2).
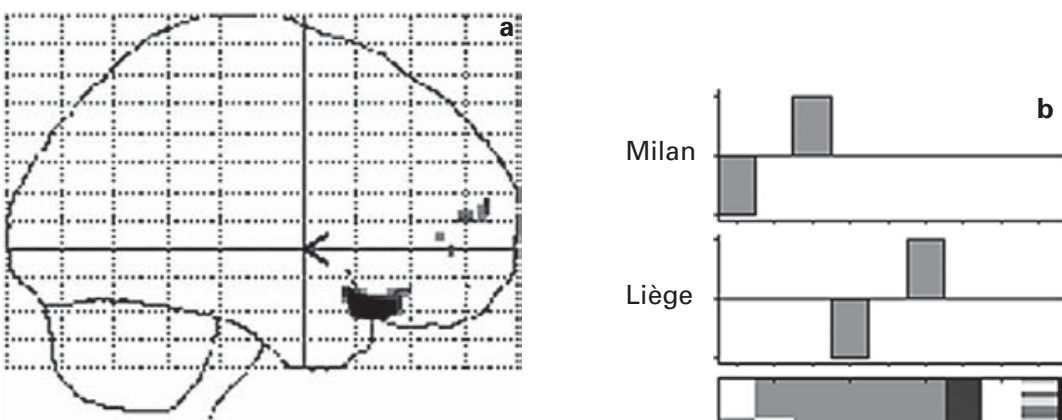

b

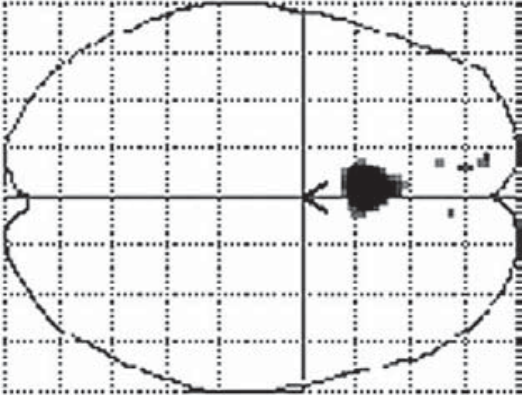

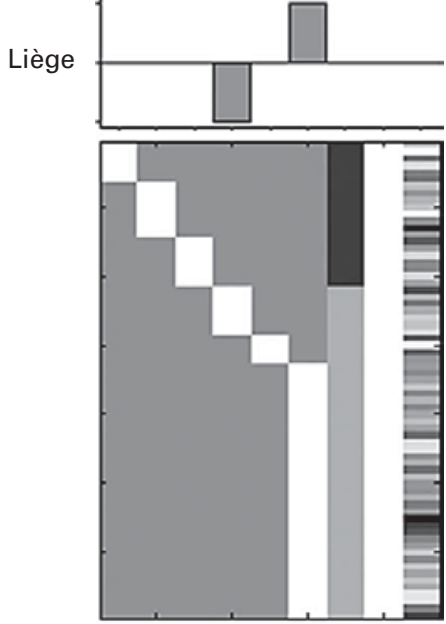

$\begin{array}{llllll}1 & 2 & 3 & 4 & 5 & 6\end{array}$
In the second analysis, we delineated, in $41 \mathrm{fv}-\mathrm{FTD}$ patients, brain regions where metabolism was correlated with the three behavioral variables. We looked for correlations between FDG-PET images and dementia severity (CDR) and NPI apathy scores, but the analyses failed to identify any significant area. However, when NPI disinhibition scores were used as the variable of interest, SPM revealed a significant correlation with the gyrus rectus $(6,28,-24)$. This pattern is illustrated in figure 1 .

For the last analysis, the conjunction revealed specific hypometabolism in the apathetic FTD group in the gyrus rectus of the orbitofrontal cortex $(4,22,-22)$, that was not shared by nonapathetic FTD subjects. This region is illustrated in figure 2 .

\section{Discussion}

The pattern of metabolic impairment observed in this prospective multicenter study of fv-FTD patients is consistent with recent neuroimaging reports. Impaired activity was observed in the anterior cingulate, ventromedial and orbital prefrontal cortex. Different areas were involved in the LPFC, including the superior and inferior frontal sulci bilaterally. The metabolism was also decreased bilaterally in a frontier area between the anterior insula and posterior LPFC. There is an overall similarity with our previous multicenter study [16], although the later was retrospective and used different diagnostic criteria for inclusion [22]. Slight differences between reports in the literature are probably related to the heterogeneity of the disease: pathological verification is very rare in neuroimaging studies, and although phenotypes may be defined with stringent diagnostic criteria [2], the limited samples of FTD patients must be heterogeneous between studies.

Correlation analyses failed to reveal any brain areas significantly correlated with dementia severity using a univariate SPM analysis. This might reflect the fact that most dementia scales, such as the CDR or MMSE [26] are inadequate to assess the deficits characterizing FTD [27]. More specifically, mixed CDR items assessing neuropsychological performance, judgment and daily functioning would not provide a consistent dementia score in FTD, because impaired activities of daily living would depend on behavioral disturbances more than on memory or orientation abilities in this disease. Thus, a heterogeneous dementia score does not appear to be related 
to any specific neural network in FTD, whereas the CDR score has been found to be related to a consistent frontoparietal 'executive' network in Alzheimer's disease [28].

The most striking clinico-metabolic relationship observed in our FTD population involved a measure of disinhibited social behavior. Our results showed that disinhibition scores were significantly correlated with a cluster of voxels located in the orbitofrontal cortex. This result is consistent with the literature revealing that disinhibited conduct is frequently observed in patients with orbitofrontal lesions $[1,29,30]$. A very recent between-groups comparison showed that metabolism in the posterior orbitofrontal cortex was impaired in FTD patients with disinhibition compared to control subjects and to FTD patients with apathy [21]. Accordingly, in the experimental setting of a reversal learning task (in which choices were associated with contingent monetary rewards and penalties), patients with damage to the orbitofrontal region were found to be unable to adjust their behavior appropriately to the contingencies of the task [31, 32]. Moreover, several neuroimaging studies have shown that this kind of task activates orbitofrontal regions in normal subjects [33-35]. These findings might be relevant to understanding the behavioral changes in FTD patients: disinhibition might correspond to an inability to adapt one's behavior to changing social rules, for example, when there is a conflict between immediate individual and delayed social reward. This is in keeping with a previous correlation observed in FTD between orbitofrontal hypometabolism and stereotypic responses with indifference to rules [20].

Finally, correlation analysis between the apathy subtest of the NPI and brain activity did not reveal significant results. This is probably due to inadequate variance in the apathy scores in our population as a whole. Moreover, apathy is a complex behavioral impairment that probably depends on different neural networks rather than on a single brain structure. Indeed, previous studies have related apathy in FTD to decreased activity in the lateral prefrontal cortices or frontopolar regions [19-21]. However, in the comparison analysis, we divided the fv-FTD patient group according to their NPI apathy scores, and the apathetic group showed specific metabolic impairment in the orbitofrontal cortex, as compared to normal controls (a result not shared by the nonapathetic FTD patients). This result is supported by a recent neuroimaging study that showed hypometabolism of anterior orbitofrontal regions (with a posterior extent overlapping with our cluster) in a group of apathetic fv-FTD subjects compared to normal controls [21]. Given the masking procedure used in our apathy comparison analysis (ensuring that hypometabolic areas associated with nonapathetic FTD were excluded from the results), it is possible that metabolic impairment in some of the previously reported regions was shared by apathetic and nonapathetic fvFTD.

Taken together, our two analyses of clinico-metabolic relationships suggest that activity in the gyrus rectus is related to both disinhibited and apathetic syndromes in fv-FTD. In keeping with the observation of a common neural correlate, Bogousslavsky et al. [36] have reported a case study of a patient with paramedian infarction of the right thalamus who showed a strong disinhibition syndrome (limited to speech), contrasted with a persistent lack of spontaneity (patient remained lying on her bed). Franceschi et al. [21] suggested that the metabolic impairment was located more in the anterior part of the orbitofrontal cortex for apathetic than for disinhibited fv-FTD patients. However, the comparison between each fv-FTD subgroup and the controls showed an overlap in the middle part of the orbitofrontal cortex. Given the overlap we also found in our analyses, it seems that orbitofrontal hypometabolism is involved in disinhibition and apathetic behaviors and that further investigations of specific networks and neurotransmitters are needed to understand how the decrease in activity in that region might induce a higher rate of various social maladjustments.

In summary, our data have confirmed that the main cerebral areas involved in fv-FTD are the medial and ventral part of the prefrontal cortex, comprising the anterior cingulate, ventromedial and orbital prefrontal cortex. In addition, we found that the gyrus rectus is significantly correlated with disinhibition scores, and is especially impaired in apathetic fv-FTD subjects. The orbitofrontal cortex is particularly important in the evaluation and updating of the emotional valence of incoming information, and this may be essential to the regulation of behavior according to social constraints.

\section{Acknowledgments}

This study was conducted on behalf of the Network for Efficiency and Standardization of Dementia Diagnosis, supported by the European Commission (5th framework) and by the EC-FP6 project Diml, LSHB-CT-2005-512146. The work in Liège is supported by grants from the FNRS, FMRE and IUAP P5/04. F.C. is a researcher at the FNRS. 


\section{References}

1 Constantinidis J, Richard J, Tissot R: Pick's disease. Histological and clinical correlations. Eur Neurol 1974; 11:208-217.

2 Neary D, Snowden JS, Gustafson L, Passant U, Stuss D, et al: Frontotemporal lobar degeneration: a consensus on clinical diagnostic criteria. Neurology 1998;51:1546-1554.

3 Hodges JR, Patterson K, Ward R, Garrard P, Bak $T$, et al: The differentiation of semantic dementia and frontal lobe dementia (temporal and frontal variants of frontotemporal dementia) from early Alzheimer's disease: a comparative neuropsychological study. Neuropsychology $1999 ; 13: 31-40$.

4 Ibach B, Poljansky S, Barta W, Koller M, Wittmann M, et al: Patterns of referring of patients with frontotemporal lobar degeneration to psychiatric in- and out-patient services. Results from a prospective multicentre study. Dement Geriatr Cogn Disord 2004; 17:269-273.

5 Bozeat S, Lambon-Ralph MA, Patterson K, Garrard P, Hodges JR: Non-verbal semantic impairment in semantic dementia. Neuropsychologia 2000;38:1207-1215.

6 Garrard P, Hodges JR: Semantic dementia: clinical, radiological and pathological perspectives. J Neurol 2000;247:409-422.

7 Chan D, Fox NC, Jenkins R, Scahill RI, Crum WR, et al: Rates of global and regional cerebral atrophy in $\mathrm{AD}$ and frontotemporal dementia. Neurology 2001;57:1756-1763.

8 Rosen HJ, Hartikainen KM, Jagust W, Kramer $\mathrm{JH}$, Reed BR, et al: Utility of clinical criteria in differentiating frontotemporal lobar degeneration (FTLD) from AD. Neurology 2002;58: 1608-1615.

9 Diehl J, Grimmer T, Drzezga A, Riemenschneider M, Forstl H, et al: Cerebral metabolic patterns at early stages of frontotemporal dementia and semantic dementia. A PET study. Neurobiol Aging 2004;25:1051-1056.

10 Rosen HJ, Gorno-Tempini ML, Goldman WP, Perry RJ, Schuff N, et al: Patterns of brain atrophy in frontotemporal dementia and semantic dementia. Neurology 2002;58:198208.

11 Galton CJ, Patterson K, Graham K, LambonRalph MA, Williams G, et al: Differing patterns of temporal atrophy in Alzheimer's disease and semantic dementia. Neurology 2001; 57:216-225.
12 Nestor PJ, Graham NL, Fryer TD, Williams GB, Patterson K, et al: Progressive non-fluent aphasia is associated with hypometabolism centred on the left anterior insula. Brain 2003; 126:2406-2418.

13 Rosen HJ, Kramer JH, Gorno-Tempini ML, Schuff N, Weiner M, et al: Patterns of cerebral atrophy in primary progressive aphasia. Am J Geriatr Psychiatry 2002;10:89-97.

14 McKhann GM, Albert MS, Grossman M, Miller B, Dickson D, et al: Clinical and pathological diagnosis of frontotemporal dementia: report of the Work Group on Frontotemporal Dementia and Pick's Disease. Arch Neurol 2001;58:1803-1809.

15 Liu W, Miller BL, Kramer JH, Rankin K, Wyss-Coray C, et al: Behavioral disorders in the frontal and temporal variants of frontotemporal dementia. Neurology 2004;62:742-748.

16 Salmon E, Garraux G, Delbeuck X, Collette F, Kalbe E, et al: Predominant ventromedial frontopolar metabolic impairment in frontotemporal dementia. Neuroimage 2003;20: 435-440.

17 Grimmer T, Diehl J, Drzezga A, Forstl H, Kurz A: Region-specific decline of cerebral glucose metabolism in patients with frontotemporal dementia: a prospective 18F-FDG-PET study. Dement Geriatr Cogn Disord 2004; 18:32-36.

18 Robert PH, Clairet S, Benoit M, Koutaich J, Bertogliati $\mathrm{C}$, et al: The apathy inventory: assessment of apathy and awareness in Alzheimer's disease, Parkinson's disease and mild cognitive impairment. Int J Geriatr Psychiatry 2002;17:1099-1105

19 Sarazin M, Pillon B, Giannakopoulos P, Rancurel G, Samson Y, et al: Clinicometabolic dissociation of cognitive functions and social behavior in frontal lobe lesions. Neurology 1998; 51:142-148.

20 Sarazin M, Michon A, Pillon B, Samson Y, Canuto A, et al: Metabolic correlates of behavioral and affective disturbances in frontal lobe pathologies. J Neurol 2003;250:827-833.

21 Franceschi M, Anchisi D, Pelati O, Zuffi M, Matarrese M, et al: Glucose metabolism and serotonin receptors in the frontotemporal lobe degeneration. Ann Neurol 2005;57:216-225.

22 Lund and Manchester Groups: Clinical and neuropathological criteria for frontotemporal dementia. The Lund and Manchester Groups. J Neurol Neurosurg Psychiatry 1994;57:416418.

23 Hughes CP, Berg L, Danziger WL, Coben LA Martin RL: A new clinical scale for the staging of dementia. Br J Psychiatry 1982;140:566572 .
24 Cummings JL, Mega M, Gray K, RosenbergThompson S, Carusi DA, et al: The Neuropsychiatric Inventory: comprehensive assessment of psychopathology in dementia. Neurology 1994;44:2308-2314.

25 Herholz K, Salmon E, Perani D, Baron JC, Holthoff V, et al: Discrimination between Alzheimer dementia and controls by automated analysis of multicenter FDG PET. Neuroimage 2002; 17:302-316

26 Folstein MF, Robins LN, Helzer JE: The MiniMental State Examination. Arch Gen Psychiatry 1983;40:812.

27 Rosen HJ, Narvaez JM, Hallam B, Kramer JH, Wyss-Coray C, et al: Neuropsychological and functional measures of severity in Alzheimer disease, frontotemporal dementia, and semantic dementia. Alzheimer Dis Assoc Disord 2004; 18:202-207.

28 Salmon E, Lespagnard P, Marique P, Peters F, Herholz K, et al: Cerebral metabolic correlates of four dementia scales in Alzheimer's disease. J Neurol 2005;252:1138.

29 Cummings JL: Frontal-subcortical circuits and human behavior. Arch Neurol 1993;50:873880 .

30 Starkstein SE, Robinson RG: Mechanism of disinhibition after brain lesions. J Nerv Ment Dis 1997; 185:108-114.

31 Rolls ET: The orbitofrontal cortex and reward. Cereb Cortex 2000;10:284-294.

32 Hornak J, O’Doherty J, Bramham J, Rolls ET, Morris RG, et al: Reward-related reversal learning after surgical excisions in orbito-frontal or dorsolateral prefrontal cortex in humans. J Cogn Neurosci 2004; 16:463-478.

33 Thut G, Schultz W, Roelcke U, Nienhusmeier M, Missimer J, et al: Activation of the human brain by monetary reward. Neuroreport 1997 ; 8:1225-1228.

34 Elliott R, Friston KJ, Dolan RJ: Dissociable neural responses in human reward systems. $\mathrm{J}$ Neurosci 2000;20:6159-6165.

35 O’Doherty J, Kringelbach ML, Rolls ET, Hornak J, Andrews C: Abstract reward and punishment representations in the human orbitofrontal cortex. Nat Neurosci 2001;4:95-102.

36 Bogousslavsky J, Ferrazzini M, Regli F, Assal $\mathrm{G}$, Tanabe H, et al: Manic delirium and frontal-like syndrome with paramedian infarction of the right thalamus. J Neurol Neurosurg Psychiatry $1988 ; 51: 116-119$ 